\title{
Global Feature Analysis and Comparative Evaluation of Freestyle In-Air-Handwriting Passcode for User Authentication
}

\author{
Duo Lu* \\ dlu@rider.edu \\ Rider University \\ Lawrence, New Jersey, USA
}

\author{
Yuli Deng \\ ydeng19@asu.edu \\ Arizona State University \\ Tempe, Arizona, USA
}

\author{
Dijiang Huang ${ }^{\dagger}$ \\ dijiang.huang@asu.edu \\ Arizona State University \\ Tempe, Arizona, USA
}

\begin{abstract}
Freestyle in-air-handwriting passcode-based user authentication methods address the needs for Virtual Reality (VR) / Augmented Reality (AR) headsets, wearable devices, and game consoles where a physical keyboard cannot be provided for typing a password, but a gesture input interface is readily available. Such an authentication system can capture the hand movement of writing a passcode string in the air and verify the user identity using both the writing content (like a password) and the writing style (like a behavior biometric trait). However, distinguishing handwriting signals from different users is challenging in signal processing, feature extraction, and matching. In this paper, we provide a detailed analysis of the global features of in-air-handwriting signals and a comparative evaluation of such a user authentication framework. Also, we build a prototype system with two different types of hand motion capture devices, collect two datasets, and conduct an extensive evaluation.
\end{abstract}

\section{CCS CONCEPTS}

- Security and privacy $\rightarrow$ Authentication; Graphical / visual passwords; Multi-factor authentication.

\section{KEYWORDS}

In-Air-Handwriting, User Authentication, Gesture Input Interface

ACM Reference Format:

Duo Lu, Yuli Deng, and Dijiang Huang. 2021. Global Feature Analysis and Comparative Evaluation of Freestyle In-Air-Handwriting Passcode for User Authentication. In Annual Computer Security Applications Conference (ACSAC '21), December 6-10, 2021, Virtual Event, USA. ACM, New York, NY, USA, 14 pages. https://doi.org/10.1145/3485832.3485906

\section{INTRODUCTION}

Gesture interfaces are generally considered the next generation of Human-Computer Interface (HCI). These novel computing platforms equipped with gesture interfaces are gaining popularity in

\footnotetext{
*The author Duo Lu is also affiliated with Arizona State University, duolu@asu.edu and this work is done when Duo Lu is a PhD student at Arizona State University.

${ }^{\dagger}$ This research is sponsored by NSF grant CNS-1925709

Permission to make digital or hard copies of all or part of this work for personal or classroom use is granted without fee provided that copies are not made or distributed for profit or commercial advantage and that copies bear this notice and the full citation on the first page. Copyrights for components of this work owned by others than ACM must be honored. Abstracting with credit is permitted. To copy otherwise, or republish, to post on servers or to redistribute to lists, requires prior specific permission and/or a fee. Request permissions from permissions@acm.org.

ACSAC '21, December 6-10, 2021, Virtual Event, USA

(c) 2021 Association for Computing Machinery.

ACM ISBN 978-1-4503-8579-4/21/12 . \$ \$15.00

https://doi.org/10.1145/3485832.3485906
}

consumer electronics [16], gaming (e.g., Sony PSVR, Facebook Oculus Quest), and education markets (e.g., Microsoft HoloLens). For example, users can play VR games and interact with virtual objects using direct hand movements captured with handheld controllers or cameras. However, on these platforms, presenting a physical keyboard or touchscreen is usually impractical, and a virtual keyboard is inconvenient to use without contact feedback. Meanwhile, many applications need to authenticate a user to unlock the device or log in to an account to access private data and services, which typically requires the user to type a password.

A freestyle in-air-handwriting passcode can be used for authentication by leveraging the native gesture user interface. Like a password-based system, the user is asked to "write a passcode string" instead of "type a password." The difference is that a password only contains typed discrete alphanumerical symbols and special characters individually distinguished. At the same time, the content of a freestyle in-air-handwriting passcode can be a string in any language, a signature, or a piece of a doodle as long as the user can naturally reproduce it but difficult to mimic by others. Meanwhile, a password is typically hashed and matched with a stored hash bit-by-bit. Still, a freestyle in-air-handwriting passcode is captured as a signal and compared with a template for authentication, in a way closer to signature verification. Thus, we call it a "passcode" rather than a "password". Besides the passcode content, the hand movement is also influenced by the writing style and the hand geometry, which provides better security and flexibility. For example, when the passcode content is leaked, such an authentication system can still defend imposters to a certain extent based on the handwriting style (like a signature but different from a password). Meanwhile, it has the desired characteristics of a password, such as changeability, revocability, and privacy. The content of the in-air-handwriting can be changed and is not necessarily linked to a person, which is different from a person biometric trait such as a fingerprint. Additionally, the coronavirus outbreak has presented new issues to the extensively deployed fingerprint and facial recognition applications. People wear facial masks and avoid touching a shared physical device, which also brings interest to gesturebased authentication methods such as freestyle in-air-handwriting passcodes.

There are three major technical challenges due to the inherent characteristics of freestyle in-air-handwriting. The first one is limited sensor capability, i.e., accurate tracking of fast hand movement with enough resolution is difficult using affordable consumer electronic motion capture sensor. Also, there is no standard on the sensor characteristics or data format. The second one is information fuzziness, i.e., there are small variations even for the same user writing the same passcode multiple times. How to tolerate these 
variations and distinguish legitimate users from imposters at the same time is not well-understood. Third, limited data availability, i.e., the authentication system can only collect a few samples at registration (typically two to five) for usability reasons. Meanwhile, collecting a dataset for developing data-driven matching algorithms is also expensive. Furthermore, it is difficult to compare different algorithms from different research groups because they usually use their own datasets. In this paper, we provide a feature analysis and comparative evaluation with an authentication framework developed by us to address these challenges. Our contributions are as follows:

1) We designed a unified model of freestyle in-air-handwriting passcode signals and implemented an authentication framework based on it. The framework supports two different types of inexpensive hand motion capture devices (a glove with an inertial sensor and a 3D stereo camera) and multiple authentication algorithms for different scenarios.

2) We collected two datasets from 180 users and 10 impostors, conducted an in-depth analysis of the in-air-handwriting signals, and compared the results with two types of sensor devices with the same group of users writing the same content for passcodes. Our datasets will be made publicly available ${ }^{1}$.

The remainder of the paper is organized as follows. Section 2 shows related works on gesture-based authentication systems, and section 3 presents the architecture of our framework. Section 4 provides an analysis of various types of features of the in-airhandwriting signal. Section 5 describes our proposed authentication algorithm. Section 6 shows the empirical evaluation results, and section 7 concludes.

\section{RELATED WORK}

As a branch of Natural User Interface (NUI) based authentication methods [27], authentication with freestyle gestures [7] generally considers two types of uses two types of devices: inertial sensors [23][15][8][11][24][2][21] or 3D cameras [31][36][35][28][5]. A few early works use dedicated devices with an accelerometer to track simple hand motions such as shake [23] or swing [15]. They demonstrate the feasibility, but they also show that these motions have limited information to distinguish different users. Instead, complicated hand movements like in-air-handwriting passcode or inair-signature can be captured using the inertial measurement unit (IMU) on a smartphone [11], or a handheld remote controller [2] for authentication. However, the behavior of writing by holding a device is different from writing with a pen. Freestyle handwriting using the fingertip in the air can be captured by a wearable data glove with an IMU [21], a smartwatch [24], or a camera-based gesture interface with the bare hand. Such cameras include the Microsoft Kinect [31][36][35], the Google Glass [28], or the Leap Motion controller [5][1][6]. Typically, the user's writing content is determined by the user, like a password or a signature, and the users are not required to move the hand to help the system convert the motion signal to discrete symbols. Hence, it is called a "freestyle inair-handwriting passcode" instead of a type of graphical password [34][30][25]. Since signals from different devices have different formats and their performance cannot be directly compared. This

\footnotetext{
${ }^{1}$ https://github.com/duolu/fmkit
}

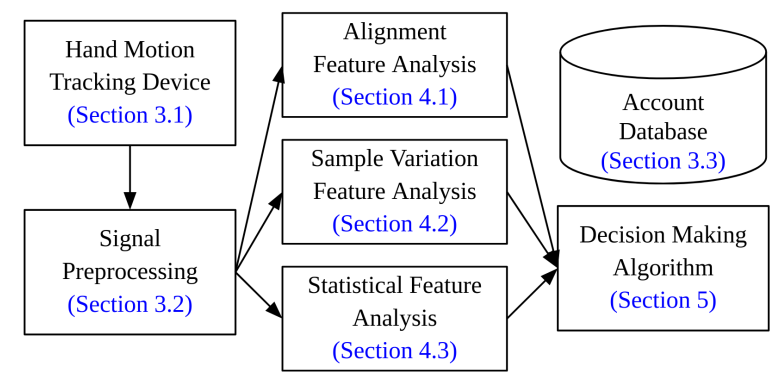

Figure 1: Authentication framework architecture.

paper proposes a unified framework that can accommodate both the inertial sensor and the Leap Motion controller. We also show their performances are comparable. Moreover, there is also a significant amount of research works in 2D online signature verification [32][13] and multitouch gesture authentication [26][37]. We do not refer to or compare with any of them because the writing behavior using a pen with the support of a surface is fundamentally different from writing using the index finger in the air.

The authentication algorithms in existing works include template matching in the temporal domain [31], comparison of frequency or statistical properties [23][38], and machine learning [5][6]. Formulating the authentication problem into a matching or binary classification problem is straightforward but existing works generally do not provide a detailed feature analysis to explain the underlying mechanism. Currently, template matching using the Dynamic Time Warp (DTW) algorithm [4] is considered the best candidate [2] due to its effectiveness, simplicity, and efficiency. With the limited amount of training data at registration, a deep neural network [20] or Hidden Markov Model (HMM) [2] may face difficulties in training, and their performance has limitations. Because matching or classification is done on signals with inherent fuzziness and noise, such an authentication system is similar to a biometric recognizer, especially an online signature verification system [12]. As a result, performance metrics for biometrics systems are generally used in the evaluation. Besides the motion signal itself, the hand geometry [10][29] can be also used to build a multiple-factor authentication system [6][14]. Fusion multiple features can be done in different levels [22] [9]. Our proposed framework supports both score-level fusion and feature-level fusion. Besides, we implemented several algorithms and compared their performance with the same dataset. We also provide an analysis of the features to explain how different design ideas work.

\section{SYSTEM MODEL}

Our proposed authentication framework consists of seven components (shown in Figure 1): a hand motion tracking device, client software for signal preprocessing, an account database, three types of feature extractors, and a decision-maker.

\subsection{Hand Motion Capture Devices}

The hand movement is captured by a Leap Motion controller [33] and a custom-made data glove with an Inertial Measurement Unit (IMU), shown in Figure 2 (c) and (d). The camera device has its 


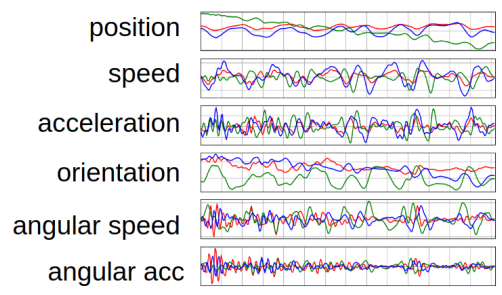

(a) signal example

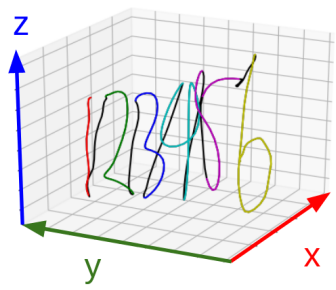

(b) trajectory example

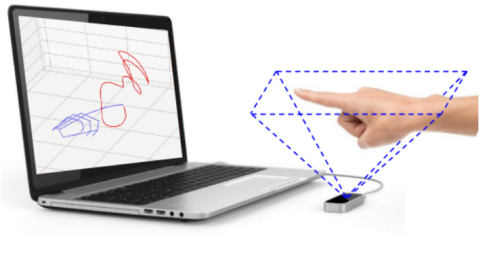

(c) the camera device

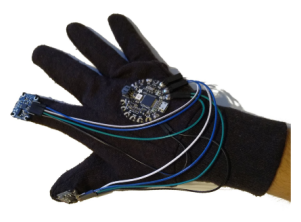

(d) the glove device

Figure 2: An example of the motion signal and the two types of hand motion capture devices.

own hand tracking software, and it can provide 3D coordinates of each joint of the hand and fingers in millimeter-level precision at around $110 \mathrm{~Hz}$. It is placed on a desk. However, we discover that the tracking results are not always reliable, especially when the hand moves quickly or when the index finger points to certain directions (e.g., if the index finger is pointing upward, it will be occluded by the palm from the perspective of the camera). Hence, the center of the palm is tracked for robustness. The glove device has a microelectromechanical IMU on the tip of the index finger, which measures acceleration and angular speed at $100 \mathrm{~Hz}$.

The captured in-air-handwriting signal is modeled as the sixdegree-of-freedom rigid body motion of a point in 3D space, including position, speed, acceleration, orientation, angular speed, and angular acceleration, in total 18 axes. With this model, both devices generate signals of the same format, enabling us to apply the same analysis methodology. An example of a signal is shown in Figure 2 (a), and the corresponding trajectory is shown in Figure 2 (b), which is obtained by tracking a user writing "123456" in the air. Note that this user deliberately writes the passcode from left to right in a legible way, and we intentionally colored each digit for illustration purposes. Most users write every letter or character at the same place very fast in an illegible way, like a signature, because it is more natural to write, and it is harder for others to mimic.

\subsection{Signal Preprocessing}

The following preprocessing steps are applied to a raw signal directly obtained from the devices:

(1) Trim the start and end when the hand does not move.

(2) Down-sample the signal to $50 \mathrm{~Hz}$ with linear interpolation.

(3) Normalize the signal reference frame. We first calculate the average position of the signal and set it as the origin. Then, we compute the average hand pointing direction using the trimmed signal and set it as the $\mathrm{x}$-axis. After that, for the camera device, we use the skeleton shape of the palm to calculate a vector pointing to the left of the palm, and set it as the $\mathrm{y}$-axis; for the glove device, we use this $\mathrm{x}$-axis and the gravity vector to set up the $\mathrm{XOZ}$ plane and find a vector perpendicular to the plane as the y-axis. Finally, we transform each sample to this new reference frame.

(4) Low-pass filter with a threshold frequency of $10 \mathrm{~Hz}$.

(5) Normalize the samples of each sensor axis to zero mean and unit variance.

The position is directly tracked for the camera device, and the orientation is estimated by the palm facing direction and hand pointing direction. The orientation is represented by three Euler angles in roll, pitch, and yaw. Higher-order states such as speed and acceleration are derived by differentiation of the position and orientation. The orientation is estimated from the angular speed for the glove device, while the position and speed are estimated by inertial dead reckoning with a regularizer. The regularizer works like a virtual attractive force to the origin to prevent unbounded drift caused by sensor bias and noise in integration. Although the obtained position does not follow the actual hand movement trajectory, it is still useful for matching because the same regularization applies to all signals.

The normalization in step 3 and step 5 are crucial since they remove the uncertainty of the hand posture relative to the sensor and the variation of the overall handwriting movement intensity. After preprocessing, a hand motion signal contains a multi-dimensional time series, denoted as a $l \times d$ matrix $R$ in general. $d$ is the number of sensor axes (18 in our case). $l$ is the signal length, i.e., the number of samples along one axis, typically from 100 to 500 . All axes have the same number of samples synchronized in time. Given a certain sampling time $i$ and a specific sensor axis $j$, the sample value is denoted as $R_{i j}$.

\subsection{Account Database and Procedures}

The account database contains a collection of accounts, and each account contains a tuple of $<\mathrm{ID}$, templates $>$. The ID is just a unique number assigned by the system to each account. The templates are generated at registration based on each feature (detailed in the "template generation" steps in sections 4 and 5).

At registration, the user must create an account by creating a passcode and writing the passcode in the air with five repetitions. A collection of five signals are generated, which is denoted as the registration signal set $\left\{R^{(1)}, R^{(2)}, \ldots, R^{(5)}\right\}$.

In this paper, we focus on the authentication problem. The user is asked to provide an account ID at login and then write a passcode in the air for authentication, similar to a password-based system. We assume the account ID is available, such as remembered by the client software or recognized by the in-air-handwriting of an ID string [20]. After finishing writing the passcode, the user keeps the hand still for one second as a termination sign to the system, and a signal is generated, denoted as the login request signal $R$. Then a set of matching algorithms process the signal $R$ and calculates a distance score $\delta$ using the templates (detailed in the "matching" steps in section 4 and section 5). After that, the authentication system makes a decision based on the score $\delta$ and a threshold 


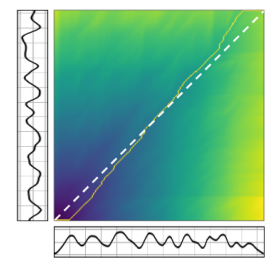

(a) warping path example

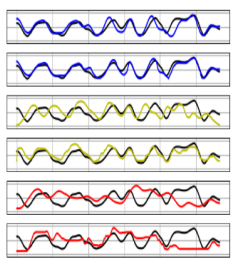

(b) alignment example

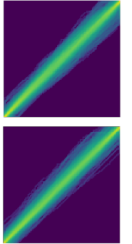

(c) same

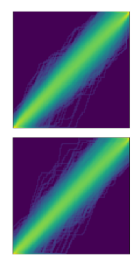

(d) collision

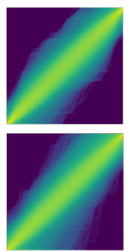

(e) diff

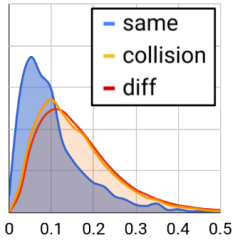

(f) distribution of $\delta_{A C}$

Figure 3: Examples of warping paths, signal alignment, and alignment analysis results. For subplot (c), (d), (e), the first row is obtained using the data from the camera device, and the second row is obtained using the data from the glove device. The same rule applies to other figures with two rows with similar content. Subplot (f) is obtained using the camera device.

(typically configured by an administrator). If the score $\delta$ is greater than the threshold, output "reject"; otherwise, output "accept".

To facilitate analysis, given a specific account $\mathrm{A}$ and a login request signal $R$, we define the class label $c$ of $R$ in three cases:

1) $c=$ same: both the request signal $R$ and the templates are generated by the owner of account A writing the same content. For example, $R$ may be obtained through legitimate user login.

2) $c=$ collision: $R$ is generated by a different user other than the owner of account A but both $R$ and the templates of account $\mathrm{A}$ are generated by writing the same content. For example, $R$ may be obtained by another user different from the legitimate user of account A, but these two users happen to choose the same passcode. Also, $R$ may be obtained by an imposter who knows the passcode content for account A.

3) $c=\operatorname{diff:} R$ and the templates of account $\mathrm{A}$ are generated by writing different passcode content, regardless of whether the same user generates them or not. For example, $R$ may be obtained by some attacker guessing the passcode of account A randomly.

Generally, in the first case, $\delta$ should be small, and if $R$ is from the legitimate user, it should be accepted. However, in the other two cases, $\delta$ should be large, and if $R$ is not from the legitimate user, it should be rejected. In practice, a system does not distinguish between case 2 and case 3, and they are only defined separately for analysis purposes. Also, a real-world system cannot see any signal in the collision class at registration. It may be able to see some signals in the diff class if there are registration signals of accounts other than account $\mathrm{A}$.

We collected the following datasets with institutional IRB approval (ASU STUDY00008279 and ASU STUDY00010539):

Dataset 1): We asked 180 users to write 360 strings, and we use them as passcodes for 360 accounts, i.e., exactly two accounts per user. Then, for each string, the user wrote it in the air five times as registration, and wrote it another five times as login requests. In total, we have 360 * 5 * 2 signals. The user determines the string content, and we asked users to avoid reusing any password they use in daily life for privacy protection. Also, we recommended that the length of an alphanumerical string should be at least eight characters, but this was not a strict restriction. A few users still created simple and short strings like "USA" or "abc123". Users might also create strings in non-Latin languages or use symbols that cannot be directly typed on a keyboard. Among all 180 users, 58 users wrote words in Chinese. The remaining 122 users wrote alphanumerical strings or strings with individual symbols (e.g., tilde, triangles, etc.). We did not have any constraints on the writing style. Most users wrote every character in the same place, like writing on an invisible vertical plane, and users generally wrote very fast in an illegible way. We did not pay incentive money to any of the users.

Dataset 2): We asked 10 impostors to write the passcode of each account in the first dataset five times. In total, we have $360{ }^{*}{ }^{*} 10$ signals. The impostors are informed of the content of the passcode, but they did not see the original account owner writing it. We paid each impostor a small amount of money for the work. Meanwhile, we collected an additional spoofing attack dataset where the impostors both know the passcode content and see the original account owner writing it. The details are shown in section 6.2.

We collected data independently using the two devices by asking the same group of users to write the identical passcode in identical environment settings. Different devices have a small influence on the user behavior, for example, the camera device restricts hand movements within its field of view, but the glove device does not have such a restriction. Roughly half of the participants are students from the authors' institute, and others are from the general public. The datasets, data collection protocol, and dataset demographical details are publicly available on our website (the URL is omitted for anonymity).

We adopt the following experiment protocol for evaluation.

Step 1) We create the templates for each account using the five registration signals in dataset 1 . We also proceed with any additional steps for registration as needed by an authentication algorithm (detailed in section 4 and section 5).

Step 2) For each account and for each of the five login request signals in dataset 1 for testing, we use it as a login request with class label $c=$ same to that account. Then, we use the signal as a login request with class label $c=$ diff to all other accounts (we assume all passcodes in our dataset are distinct).

Step 3) For each account and for each of the fives login request signals in dataset 1 for testing, use it as a login request with class label $c=$ same to that account. Then, for each signal generated by impostors in dataset 2 , use it as a login request with class label $c=$ collision to the target account.

If a request signal with class label $c=$ same is rejected, it is a False Negative (FN); otherwise, it is a True Positive (TP). If a request signal with class label $c=$ diff or $c=$ collision is accepted, it is a False Positive (FP); otherwise, it is a True Negative (TN). The results generated in this step are denoted as "results without collision", 
while the results generated in this step are denoted as "results with collision". The same user may reuse the same passcode for multiple accounts in practice, and different users may happen to use the same passcode. Hence, the actual performance can be estimated somewhere between these two cases.

\subsection{Applicability}

The target application scenarios of our framework are mainly VR/AR headsets and wearable computing platforms that already have a gesture interface but lack a keyboard or a touchscreen. Other application scenarios may also benefit from our framework, e.g., scenarios that prefer touchless gesture interfaces for a level of cleanliness. Our framework can be used for two different purposes. The first one is online user login, where the server is remotely connected to the client via the Internet. For example, the user can sign into an online personal account through the gesture interface on a VR headset. The second one is local user login, where the client and server reside on the same machine. For example, the user can unlock his or her wearable devices through the gesture interface. In addition, our framework can also be used as a supplementary authentication factor in a Multi-Factor Authentication (MFA) system.

Our framework can be implemented with similar server infrastructures using password-based login because on the server side only software changes are required. The requirement of the network between the client and the server for the remote user login scenario is similar to existing web applications through a secure communication channel over SSL/TSL with Public Key Infrastructure (PKI).

In this paper, we make the following security assumptions: (1) the device on the user side is secure(i.e., no sniffing backdoor); (2) the storage and computing infrastructure of the authentication server is secure (i.e., the server program will not leak the templates to others. In reality, server storage leakage should be considered and template protection mechanisms are discussed in a separate work [18] by us); and (3) the communication channel between the user and the server is secure. Based on the assumptions, in this paper we are mainly interested in random guessing attacks and spoofing attacks generated by human imposters on the user side. The attacker's goal is to sign into the victim's account or be identified as the victim. If the attack is successful, the account owner may suffer from loss of the account or leakage of private information. These two types of attacks are studied experimentally with the step 2 and step 3 of the experiment protocol explained in section 3.3.

\section{FEATURE ANALYSIS}

In this section, we analyze the signal variation in time (section 4.1), sample magnitude (section 4.2), and statistical features (section 4.3).

\subsection{Alignment Analysis}

A signal $R$ can be aligned to another signal $T$ by Dynamic Time Warping (DTW) [4] to generate an aligned signal $S$. Here $T$ is called a template. Specifically, we first run DTW on $R$ and $T$ with all sensor axes and a window constraint of \pm 50 samples. The distance function in DTW between two individual samples is the Euclidean distance. The warping path is shown in Figure 3 (a) as the solid line on the left image, which indicates the writing behavior variation in time. If there is no variation between the two signals, it should be a straight diagonal line as the dashed line. This warping path indicates the correspondences between samples in $R$ and $T$. Given the path, we calculate each sample of the aligned signal $S_{i j}$ by taking the average of those samples of $R$ which are mapped to $T_{i j}$ on the warping path.

This alignment step can be applied to any pair of signals, regardless of whether they are generated by writing the same content or not. After alignment, $S$ and $T$ will have the same length (denoted as $l$ ). Examples are shown in Figure 3 (b), where the black signal is $T$ and the colored signals are $R$ (without alignment) or $S$ (with alignment). An illustration of the warping path distribution is shown in Figure 3(c) (d), and (e), which is obtained by overlaying warping paths of 1,000 pairs of signal and template in different classes. This indicates signals of different classes are "warped" in different intensities. Especially, the signals generated by the same user writing the same content have very similar shapes. With this observation, the following DTW matching algorithm is designed as a baseline algorithm.

Template Generation: Given $m$ raw signals at registration for an account, first preprocess and align them to the first signal to obtain $m$ aligned signals $\left\{S^{(1)}, S^{(2)}, \ldots, S^{(m)}\right\}$. Then calculate $T$ as follows (essentially element-wise average),

$$
T_{i j}=\frac{1}{m} \sum_{k}^{m} S_{i j}^{(k)},
$$

where $T$ is stored as the template in the account database.

Matching: Given an account with a template $T$, and an authentication request signal $R$, first preprocess $R$ and align it to $T$ to obtain an aligned signal $S$. Then calculate the element-wise distance $D_{i j}=\left|T_{i j}-S_{i j}\right|$; finally calculate the distance,

$$
\delta_{D T W}=\frac{1}{l d} \sum_{i}^{l} \sum_{j}^{d} D_{i j} .
$$

Here, $l$ is the template length, $d$ is the number of sensor axes. $\delta_{D T W}$ can be compared with the threshold directly for making a decision, or it can be considered as a synthesized feature in the fusion methods.

We define another distance score called the alignment cost (denoted as $\delta_{A C}$ ). Formally, assuming the segment between $i_{s}^{\prime}$ and $i_{e}^{\prime}$ of $R$ is aligned to the $i$ th sample of $T$,

$$
\delta_{A C}=\frac{1}{l} \sum_{i=1}^{l}\left(i-\frac{i_{s}^{\prime}+i_{e}^{\prime}}{2}\right) .
$$

The distribution of $\delta_{A C}$ is shown in Fig 3 (f). This alignment cost is a synthesized feature with relatively weak discriminative capability but can be used in the fusion methods.

\subsection{Sample Variation Analysis}

The DTW matching each sample is treated equally, which is reasonable for alignment but not necessarily for matching. We propose the following Threshold-Then-Vote (TTV) algorithm based on the DTW matching with improvements. This algorithm is originally designed only for the camera device using a different signal format in our previous work [19]. In this paper we revise it and show that it is applicable to signals obtained from both types of sensors. 


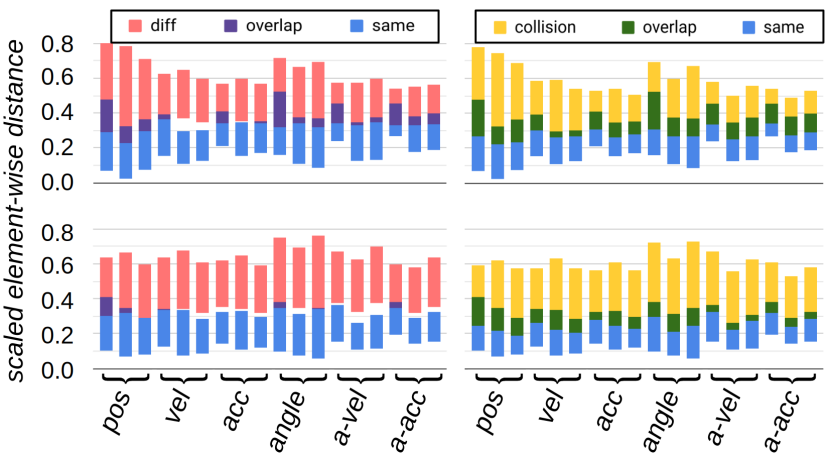

Figure 4: $\pm 2 \sigma$ ranges of the aggregated scaled element-wise distance $\frac{1}{l} \sum_{i}^{l} D_{i j}^{\prime}$ respect to the sensor axes for different classes and devices. The left figure shows results with $c=$ same and $c=$ diff, and the right figure shows results with $c=$ same and $c=$ collision.

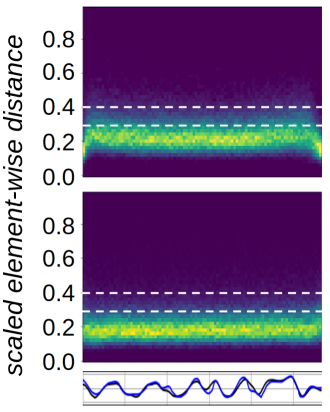

(a) same (b) collision

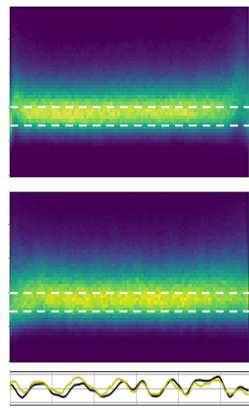

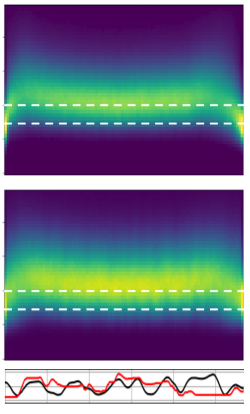

(c) diff
Figure 5: Distribution of the scaled element-wise distance $\frac{1}{d} \sum_{j}^{d} D_{i j}^{\prime}$ respect to the time for different classes. The horizontal axis is the time and signals are temporally normalized to the same length. The brightness indicates the density. The two dashed lines are the $t h_{1}$ and $t h_{2}$.

Template Generation: Calculate the template $T$ in the same way as the DTW matching algorithm. Additionally, calculate the template uncertainty $C$ as follows,

$$
C_{i j}=\operatorname{var}\left(S_{i j}^{(1)}, S_{i j}^{(2)}, \ldots, S_{i j}^{(m)}\right),
$$

where the $\operatorname{var}()$ function calculates the variance. Both $T$ and $C$ are stored in the account database.

Matching: Given an account with $T, C$, and request signal $R$, first preprocess $R$ and align it to $T$, and calculate element-wise distance $D_{i j}$ in the same way as the DTW matching; then multiply $D_{i j}$ by two scaling factors $P_{i j}$ and $Q_{i j}$ derived from the template value $T$ and variance $C$ (explained later in this subsection); finally calculate:

$$
\delta_{T T V}=\frac{1}{l} \sum_{i}^{l} \operatorname{round}\left[\frac{1}{d} \sum_{j}^{d} \operatorname{TTV}\left(D_{i j} * P_{i j} * Q_{i j}\right)\right] .
$$

Similar to $\delta_{D T W}, \delta_{T T V}$ can be used directly for decision making or as a synthesized feature to be fused with other features. The round () function means rounding to the nearest integer (either 0 or 1 in our case), which is essentially a majority vote. $\operatorname{TTV}(x)$ is the same as that in our previous work [19], i.e., if $D_{i j} * P_{i j} * Q_{i j}$ is over the threshold $t h_{2}$, it is set to 1 ; if $D_{i j} * P_{i j} * Q_{i j}$ is below the threshold $t h_{1}$, it is set to 0 ; Otherwise, it is set to 0.5 .

This function maps an element-wise distance to an element-wise decision as "match" (i.e., 0), "non-match" (i.e., 1), or "unsure" (i.e., 0.5). Then the element-wise decisions are aggregated, and the portion of non-match votes is the distance score. Here $t h_{1}$ and $t h_{2}$ are tunable parameters determined empirically (in our case, $t h_{1}=0.3$, $t h_{2}=0.4$, explained later). When $S$ and $T$ are obtained from the same user writing the same passcode, because of slight handwriting behavior change or misalignment, such local non-matches exist, but their votes are only a few. However, when $S$ and $T$ are obtained from different users or from writing different contents, they are different in many places along multiple axes, and the non-matches dominates.

The two scaling factors $P$ and $Q$ are also proposed in our previous work [19] for only one type of device. In this paper, we show that it is applicable to the signals obtained from both types of sensors. The design philosophy is explained as follows. Basically, the element-wise distance varies differently in different places and along different sensor axes. The general observation is that $D_{i j}$ is large when $T_{i j}$ and $C_{i j}$ are large. Rather than varying the thresholds $t h_{1}$ and $t h_{2}$, the scaling of element-wise distance is introduced to apply less importance to some samples if the template has a larger value or a larger variance at those places. $P_{i j}$ and $Q_{i j}$ are calculated as follows (same as that in our previous work [19]):

$$
P_{i j}=1 /\left(1+p * T_{i j}\right), Q_{i j}=1 /\left(1+q * C_{i j}\right),
$$

where $p$ and $q$ are tunable parameters determined empirically (in our experiments, $p=0.5, q=1.0)$. Basically, $p$ and $q$ are chosen such that $D_{i j}$ is roughly constant for most of $R$ with $c=$ same. Now we provide more analysis on the element-wise distance. For convenience, we denote $D_{i j} * P_{i j} * Q_{i j}$ as $D_{i j}^{\prime}$.

First, in Figure 4, we show the distribution of $D_{i j}^{\prime}$ respect to the sensor axes averaged along the time, i.e., an approximation of $p\left(\frac{1}{l} \sum_{i}^{d} D_{i j}^{\prime} \mid c\right)$. This shows the signal variation in each sensor axis, and larger overlap indicates more difficulty in distinguishing different classes based on that axis. Since the writing behavior in the air is similar to writing on an invisible wall, and in the posture normalization preprocessing step, the $\mathrm{x}$-axis is defined as the averaged pointing direction (i.e. against the wall), the uncertainty in that direction is larger. Also, the position axes show a larger variation range, which means users are generally better at keeping the same pattern in speed and acceleration than maintaining the same trajectory. We believe that hand movement speed and acceleration are directly related to the force generated by the muscle using "muscle memory" while maintaining position and trajectory requires visual feedback and attention from the brain. Since handwriting is an acquired skill, the "muscle memory" is a behavioral biometric trait and relatively stable.

Second, in Figure 5, we show the distribution of $D_{i j}^{\prime}$ respect to the time averaged over all sensor axe, i.e., an approximation of $p\left(\frac{1}{d} \sum_{j}^{d} D_{i j}^{\prime} \mid c\right)$. For those signal and template generated by the same user writing the same content $(c=s a m e)$, the average of $D_{i j}^{\prime}$ 
is generally smaller than those generated by different users writing the same content $(c=$ collision) or by writing different contents $(c=$ diff). This is expected. There is an overlap in a region (shown as the dashed horizontal lines), which determines the two parameters $t h_{1}$ and $t h_{2}$. Generally, below $t h_{1}$, it is desired that

$$
p\left(\frac{1}{d} \sum_{j}^{d} D_{i j}^{\prime} \mid c=\text { same }\right)>p\left(\frac{1}{d} \sum_{j}^{d} D_{i j}^{\prime} \mid c=\text { collision }\right),
$$

and above $t h_{2}$, it is desired that

$$
p\left(\frac{1}{d} \sum_{j}^{d} D_{i j}^{\prime} \mid c=\operatorname{diff}\right)>p\left(\frac{1}{d} \sum_{j}^{d} D_{i j}^{\prime} \mid c=\text { collision }\right) .
$$

From Figure 4 and 5, it can be observed that samples in different sensor axes and at different time should be considered with different importance (i.e., using different weight). Also, in Figure 5, it can be observed that the distribution of sample differences does not change along the time given a large number of accounts with diverse passcode contents. However, the local non-matches of signals from different accounts vary in different ways. Hence, a per-account decision-making algorithm can perform better than a global one, explaining the performance gain detailed in section 7. Additionally, there are small anomalies on both ends since users usually raise their hands to start the writing and put down the hand to terminate the writing. Hence, the performance can be improved slightly by trimming the start and the end again after alignment.

\subsection{Statistical Feature Analysis}

In this section, we investigate those statistical aspects of the signal which are lost in the alignment and normalization steps. Specifically, the following statistical features are studied.

1) The mean of each sensor axis across time $\mathbf{M}=\left(\mu_{1}, \ldots, \mu_{d}\right)$, where $\mu_{j}=\operatorname{mean}\left(R_{1 j}, \ldots, R_{l j}\right)$.

2) The standard deviation of each sensor axis across time $\Sigma=$ $\left(\sigma_{1}, \ldots, \sigma_{d}\right)$, where $\sigma_{j}=\operatorname{std}\left(R_{1 j}, \ldots, R_{l j}\right)$.

3) The correlation coefficients between pairs of adjacent sensor axes $\mathbf{P}=\left(\alpha_{x y}, \alpha_{y z}, \alpha_{x z}, \beta_{x y}, \beta_{y z}, \beta_{x z}, \ldots\right)$, where $\alpha_{x y}, \alpha_{y z}, \alpha_{x z}$ are the correlation of the three axes of position, $\beta_{x y}, \beta_{y z}, \beta_{x z}$ are the correlation of the three axes of speed, and so on. Here,

$$
\alpha_{x y}=\frac{\sum_{i}\left(R_{i x}-\mu_{x}\right)\left(R_{i y}-\mu_{y}\right)}{\sigma_{x} \sigma_{y}} .
$$

4) Sum of amplitude of each axis across time $\Lambda=\left(\lambda_{1}, \ldots, \lambda_{d}\right)$, where $\lambda_{j}=\sum_{i=1}^{l}\left|R_{i j}\right|$.

5) Portion of low frequency components $\mathbf{L}=\left(\xi_{1}, \ldots, \xi_{d}\right)$, where $\xi_{j}=$ mean $\left(l o w \_p a s s\left(\operatorname{FFT}\left(\left(R_{1 j}, R_{2 j}, \ldots, R_{l j}\right)\right)\right)\right)$. The FFT() function computes the Fast Fourier transform coefficients on the samples of a specific sensor axis. The low_pass() function zeros the coefficients higher than $3 \mathrm{~Hz}$.

Collectively, the statistical feature vector of a signal $R$ is the combination of them, defined as $f(R)=(\mathbf{M}, \Sigma, \mathbf{P}, \boldsymbol{\Lambda}, \mathbf{L})$, where $f(R)$ is the statistical feature extractor. In total, there are $18{ }^{*} 5$ individual elements. Some of the features are used in our previous work [21] for only one type of sensor.

Template Generation: Given $\left\{R^{(1)}, R^{(2)}, \ldots, R^{(m)}\right\}$ as the $m$ signals at registration for an account, first run the prepossessing step 1 to 3 for each signal, extract statistical features, then run the preprocessing step 4 and 5 , and finally calculate the element-wise mean

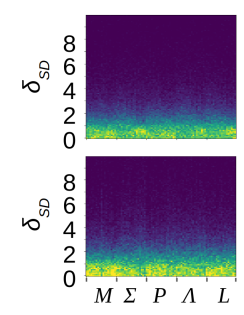

(a) same

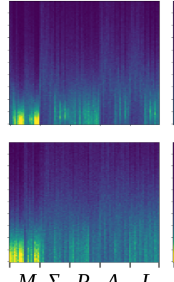

(b) collision

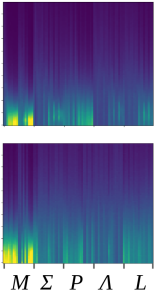

(c) diff

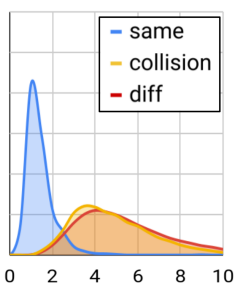

(d) distribution of $\delta_{S D}$
Figure 6: Distribution of statistical difference for different classes. The sequence of sensor axes within a specific type of statistical features is the same as Figure 4. Subplot (d) is obtained using the camera device.

$\boldsymbol{\mu}_{S F}$ and standard deviation $\boldsymbol{\sigma}_{S F}$ as templates:

$$
\begin{gathered}
\boldsymbol{\mu}_{S F}=\operatorname{mean}\left(f\left(R^{(1)}\right), f\left(R^{(2)}\right), \ldots, f\left(R^{(m)}\right)\right), \\
\boldsymbol{\sigma}_{S F}=\operatorname{std}\left(f\left(R^{(1)}\right), f\left(R^{(2)}\right), \ldots, f\left(R^{(m)}\right)\right) .
\end{gathered}
$$

Matching: Given an account with template $\boldsymbol{\mu}_{S F}$ and $\sigma_{S F}$, and an authentication request signal $R$, run the same preprocessing steps as those in the template generation, then calculate the following aggregated statistical difference score:

$$
\delta_{S D}=\operatorname{mean}\left(\operatorname{abs}\left(f(R)-\boldsymbol{\mu}_{S F}\right) / \boldsymbol{\sigma}_{S F}\right),
$$

where the subtraction and division are all element-wise, the mean () function averages all elements of its vector input. The idea behind this statistical difference is the same as the Mahalanobis distance.

The distribution of each component of the statistical difference vector $a b s\left(f(R)-\boldsymbol{\mu}_{S F}\right) / \boldsymbol{\sigma}_{S F}$ for different classes is shown in Figure 6 (a), (b) and (c). Also, the distribution of $\delta_{S D}$ is shown in Figure 6 (d). They are generally weak features, especially the mean of most sensor axes, such as the speed and acceleration, because many of them tend to be around zero. Additionally, signal variance, amplitude, and low frequency components are strongly correlated, because all these three features will have a larger magnitude if the user writes intensively. As a result, we argue that removing them in the preprocessing step does not incur much loss of information for user authentication.

We incorporate two additional statistical features as discussed in our previous work [19] using only one type of sensor. The first one is the difference of lengths between a signal $R$ and a template $T$, denoted as $\delta_{L D}$. It is calculated as $\delta_{L D}=\left|l_{R}-l_{T}\right| / l_{T}$, where $l_{R}$ and $l_{T}$ are the lengths of the signal $R$ and template $T$ respectively. The second one is the hand geometry difference, denoted as $\delta_{H G D}$. It is the average length difference of finger bone segments in ratio. It is computed as $\delta_{H G D}=\operatorname{mean}\left(\operatorname{abs}\left(\mathbf{h}_{R}-\mathbf{h}_{T}\right) / \mathbf{h}_{T}\right)$, where $\mathbf{h}_{R}$ and $\mathbf{h}_{T}$ are the vectors consisting of the length of finger bone segments measured by the camera device. The glove device cannot obtain this feature. More details about the hand geometry feature are provided in our previous work [19]. Similar to the alignment cost, these two features are weak but they can be used in the fusion process.

\section{AUTHENTICATION ALGORITHM}

We propose two authentication algorithms based on our feature analysis: Score-Fusion (section 5.1) and Feature-Fusion (section 5.2). 


\subsection{Score Fusion}

The Score Fusion (S-Fusion) scheme uses a weighted average of the matching scores on each type of feature.

Template Generation: Given the registration signals, generate templates for the temporal, statistical, and hand geometry features.

Matching: Run the matching algorithm of each feature and calculate the score:

$$
\begin{aligned}
\delta_{\text {other }}= & w_{1} \delta_{A C}+w_{2} \delta_{S D}+w_{3} \delta_{L D}+w_{4} \delta_{H G D}, \\
& \delta_{S-\text { Fusion }}=\delta_{T T V}+\delta_{\text {other }},
\end{aligned}
$$

where $\delta_{T T V}$ is the matching score using the TTV algorithm (i.e., the temporal distance), $\delta_{A C}$ is the alignment cost score, $\delta_{S D}$ is the statistical difference score, $\delta_{H G D}$ is the hand geometry difference score, $w_{1}$ to $w_{4}$ are tunable parameters (in our case, $w_{1}=0.1, w_{2}=$ $\left.0.03, w_{3}=0.3, w_{4}=0.4\right) . \delta_{T T V}$ is considered as the primary feature with strong discriminative capability, and the others are supplemental features. The glove device is unable to measure hand geometry and $w_{4}$ is always zero.

These weights $w_{1}$ to $w_{4}$ are determined empirically. In our case, as shown in Figure 7, we first plot the supplemental scores concerning the primary score with 1,000 signals in each class and then manually draw a linear decision boundary. The weights are computed by the inverse of the slopes of these lines. This is designed mainly for simplicity, and it is not necessarily optimal. More sophisticated score fusion schemes [22] [9] can be employed with a density estimation of the scores to improve performance.

\subsection{Feature Fusion}

If we inspect each individual element-wise distance $D_{i j}$, it is essentially a type of weak feature. Inspired by the idea of the ensemble of weak features using a weighted average, we design a Feature Fusion (F-Fusion) scheme as follows.

Template Generation: Same as Score Fusion.

Matching: Align the signal $R$ to $T$ and calculate the score:

$$
\begin{gathered}
\delta_{T-F u s i o n}=b+\sum_{i=1}^{l} \sum_{j=1}^{d} w_{i j} D_{i j}, \\
\delta_{\text {F-Fusion }}=\delta_{T-F u s i o n}+\delta_{\text {other }} .
\end{gathered}
$$

In this scheme, each feature has its own weight $w_{i j}$, and since they vary differently, the $w_{i j}$ should be calculated based on the data for each account. $b$ is a bias to keep the score between 0 and 1 . DTW matching is a special case of T-Fusion by setting all weights to the same value. TTV sets the weights systematically based on a few hyperparameters with additional nonlinear cutoff. Determining the weights $w_{i j}$ is essentially fitting a decision plane in the feature space, and this can be done for each account independently. In our case, these weights $w_{i j}$ as well as $w_{1}$ to $w_{4}$ are learned from the data at registration using a soft margin Support Vector Machine (SVM) in a per account manner. Specifically, at registration, for each account, we use the $D_{i j}$ calculated by five signals generated by the account owner as positive samples, and we use the $D_{i j}$ calculated by 1,000 signals randomly selected from other accounts as negative samples to train an SVM. One limitation of this method is that at registration, data from all accounts must be accessed. This is possible for an online application with many accounts but infeasible for device unlock scenarios with only one or a few users.

\section{EXPERIMENTAL EVALUATION}

We build a prototype system and evaluate all matching algorithms mentioned in previous sections with the datasets and protocol detailed in Section 3.3. Due to the imbalance of positive and negative testing data, the main performance metrics are False Accept Rate (FAR) and False Reject Rate (FRR). Specifically, FAR = \#\{FP $\} /(\#\{F P\}$ $+\#\{\mathrm{TP}\})$, and $\mathrm{FRR}=\#\{\mathrm{FN}\} /(\#\{\mathrm{FN}\}+\#\{\mathrm{TN}\})$, where \#\{\} means the number of FP, TP, FN, or TN. FAR and FRR can change by varying the decision threshold, and Equal Error Rate (EER) is the value where FRR is equal to FAR. The results are shown in Table 1. Here FAR1K and FAR10K indicate the FRR when FAR is $10^{-3}$ and $10^{-4}$, respectively. ZeroFAR is the smallest FRR when FAR is zero, and ZeroFRR is the smallest FAR when FRR is zero. In the table, the row DTW(2) and TTV(2) show the results using only the first two signals at registration for template construction. We also show the modified Receiver Operating Characteristic (ROC) curves in Figure 8 by plotting the FAR against the FRR instead of plotting the True Accept Rate (TAR) against the FRR. Compared to the original ROC curves, these modified ROC curves look better in log-log scale.

These matching algorithms have a gradual improvement in performance with an increase in the number of parameters. The DTW algorithm is the simplest, with no need for parameter tuning. The TTV algorithm requires to determine four parameters $\left(p, q, t h_{1}, t h_{2}\right)$, and the S-Fusion algorithm requires another four ( $w_{1}$ to $\left.w_{4}\right)$, which can be set up with a small pilot dataset or with rule-of-the-thumb values provided in this paper. These methods can also work with only one or a few accounts in the account database, e.g., scenarios such as unlocking a device. In T-Fusion and F-Fusion, the numbers of parameters are roughly the same as an additional template, and they are learned from data in the account database. These two methods can be used in online login.

The limitation of all proposed methods is essentially the quality of the template, i.e., it is difficult to use one template generated from the limited number of signals at registration to capture the whole picture of the user's handwriting behavior variation. To mitigate this limitation, we conduct two additional experiments, and the results are shown in the last two rows of Table 1. For TFusion(A), we augment the five registration signals to 125 signals by performing a slight random rotation, adding a small perturbation, and swapping a random segment given a randomly picked pair of training signals. Then, we use the augmented signals to train the SVM. The idea is to artificially create signal variation so that the learned model may be more robust. For T-Fusion(E), we use each of the five registration signals as a template, train five individual SVM models. We take the minimum of the five scores generated by the five models as the ensemble score during the testing. The idea is to use multiple templates instead of one. This idea of matching the login request to each of the registration signals and choosing the best result can also be applied to the DTW method and the TTV method. These methods show further improvements.

A comparison to existing works using in-air-handwriting is shown in Table 2 . Their performance cannot be compared directly because each work has its own dataset collected with a different type of device and in an environment setting unique to that work. Moreover, their datasets are not openly available. We list them in this paper as a general reference. Readers should also be careful 

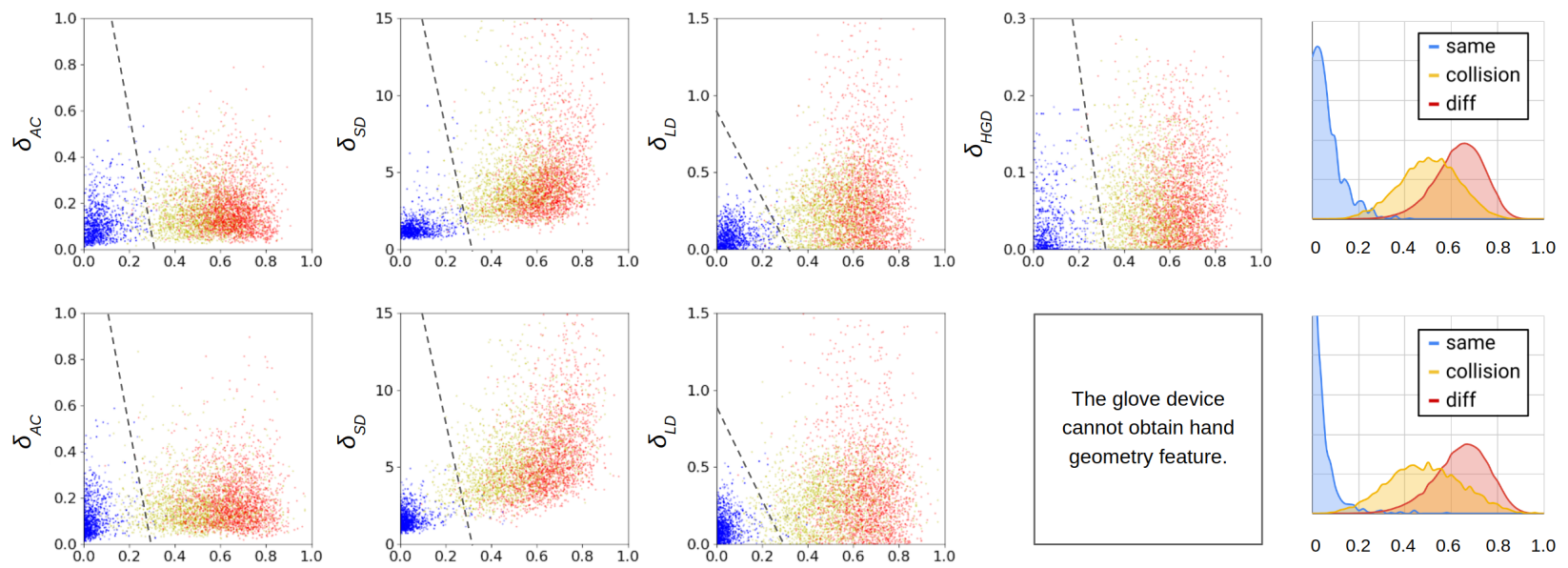

(a) $\delta_{A C}$ VS. $\delta_{T T V}$

(b) $\delta_{S D} v S . \delta_{T T V}$

(c) $\delta_{S D} v S . \delta_{T T V}$

(d) $\delta_{H G D}$ vs. $\delta_{T T V}$

(e) distribution of $\delta_{T T V}$

Figure 7: Distribution of the scores from various features.

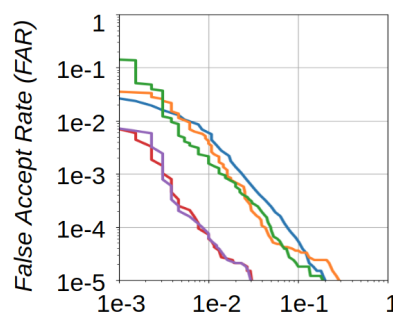

(a) w/o collision, camera

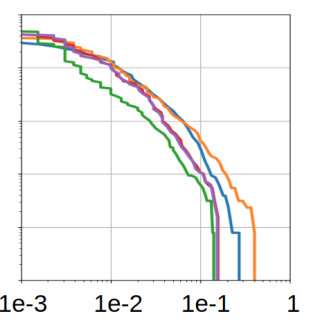

False Reject $R$

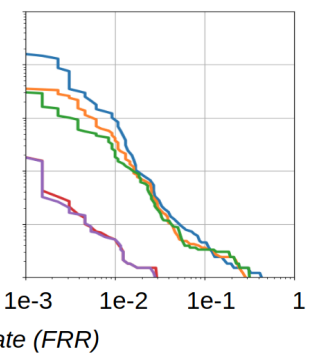

(c) w/o collision, glove

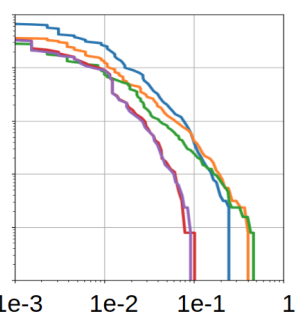

(d) $w /$ collision, glove

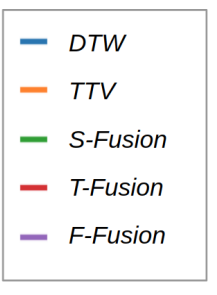

(b) w/ collision, camera

Figure 8: Modified Receiver Operating Characteristic (ROC).

Table 1: Authentication performance results.

\begin{tabular}{|c|c|c|c|c|c|c|c|c|c|c|c|c|c|c|c|c|}
\hline \multirow{3}{*}{ method } & \multicolumn{8}{|c|}{ using data from the camera device } & \multicolumn{8}{|c|}{ using data from the glove device } \\
\hline & \multicolumn{5}{|c|}{ without collision (in \%) } & \multicolumn{3}{|c|}{ with collision (in \%) } & \multicolumn{5}{|c|}{ without collision (in \%) } & \multicolumn{3}{|c|}{ with collision (in \%) } \\
\hline & EER & $\begin{array}{c}\text { FAR } \\
1 \mathrm{~K}\end{array}$ & $\begin{array}{c}\text { FAR } \\
10 \mathrm{~K}\end{array}$ & $\begin{array}{l}\text { Zero } \\
\text { FAR }\end{array}$ & $\begin{array}{l}\text { Zero } \\
\text { FRR }\end{array}$ & EER & $\begin{array}{c}\text { FAR } \\
1 \mathrm{~K}\end{array}$ & $\begin{array}{l}\text { Zero } \\
\text { FAR }\end{array}$ & EER & $\begin{array}{c}\text { FAR } \\
1 \mathrm{~K}\end{array}$ & $\begin{array}{c}\text { FAR } \\
10 \mathrm{~K}\end{array}$ & $\begin{array}{l}\text { Zero } \\
\text { FAR }\end{array}$ & $\begin{array}{l}\text { Zero } \\
\text { FRR }\end{array}$ & EER & $\begin{array}{c}\text { FAR } \\
1 \mathrm{~K}\end{array}$ & $\begin{array}{l}\text { Zero } \\
\text { FAR }\end{array}$ \\
\hline DTW(2) & 1.24 & 5.75 & 17.69 & 71.40 & 28.76 & 5.10 & 27.6 & 37.2 & 1.24 & 3.80 & 10.52 & 49.46 & 98.18 & 5.04 & 24.0 & 38.9 \\
\hline TTV(2) & 1.00 & 4.81 & 12.18 & 44.57 & 16.49 & 3.64 & 19.9 & 37.2 & 1.12 & 2.97 & 7.30 & 48.84 & 50.97 & 4.17 & 23.0 & 38.9 \\
\hline DTW & 0.81 & 2.39 & 7.56 & 56.36 & 25.00 & 3.08 & 13.3 & 29.5 & 0.75 & 1.33 & 5.26 & 43.80 & 8.56 & 2.95 & 12.0 & 19.6 \\
\hline TTV & 0.70 & 2.38 & 7.99 & 23.64 & 15.54 & 2.16 & 15.2 & 34.8 & 0.68 & 0.85 & 2.86 & 35.50 & 11.23 & 2.39 & 13.6 & 23.6 \\
\hline S-Fusion & 0.50 & 1.38 & 4.95 & 37.75 & 42.38 & 1.83 & 7.4 & 15.4 & 0.39 & 0.93 & 2.06 & 36.12 & 16.21 & 1.98 & 15.6 & 30.9 \\
\hline T-Fusion & 0.22 & 0.32 & 0.78 & 12.33 & 7.35 & 2.61 & 10.9 & 17.0 & 0.16 & 0.16 & 0.39 & 2.95 & 6.57 & 1.51 & 4.7 & 6.2 \\
\hline F-Fusion & 0.26 & 0.31 & 0.85 & 12.00 & 7.30 & 2.61 & 10.7 & 16.6 & 0.16 & 0.16 & 0.39 & 3.10 & 4.25 & 1.51 & 4.4 & 5.6 \\
\hline T-Fusion(A) & 0.21 & 0.23 & 0.57 & 10.62 & 3.23 & 2.74 & 10.6 & 23.2 & 0.16 & 0.16 & 0.42 & 2.79 & 0.50 & 1.86 & 6.5 & 12.2 \\
\hline T-Fusion(E) & 0.10 & 0.12 & 0.78 & 10.70 & 4.72 & 2.40 & 8.91 & 18.3 & 0.16 & 0.16 & 0.39 & 2.02 & 2.29 & 1.38 & 3.5 & 5.1 \\
\hline
\end{tabular}

to interpret the numbers in Table 1 and Table 2 because they may have some level of uncertainty due to the limited number of false rejects in the experiments. Meanwhile, different signal preprocessing implementations can also have small influences in these results. Moreover, we intentionally allow each user to have two strings in our datasets to reveal more "hard cases" for analysis since we believe there are always users with similar writing styles. The goal of this research is not trying to design an authentication algorithm with the best performance, but discussing the feasibility and design space of authentication algorithms as well as obtaining insights through global feature analysis of the signals.

It is interesting to see that both the camera device and the glove device share a lot in common in the characteristics of the features and the authentication performances, even though these 
Table 2: Comparison to existing works.

\begin{tabular}{|l|c|c|c|c|c|}
\hline Ref. & $\begin{array}{c}\text { number } \\
\text { of users }\end{array}$ & $\begin{array}{c}\text { EER } \\
\text { (w/o collision) }\end{array}$ & $\begin{array}{c}\text { EER } \\
\text { (w/ collision) }\end{array}$ & Device & Algorithm \\
\hline Ours (camera) & 180 & $0.10 \% \sim 1.24 \%$ & $1.83 \% \sim 5.10 \%$ & Leap Motion & SVM, TTV, DTW \\
\hline Ours (glove) & 180 & $0.16 \% \sim 1.24 \%$ & $1.38 \% \sim 5.04 \%$ & custom data glove & SVM, TTV, DTW \\
\hline Liu et al.[17] & $20 \sim 25$ & $\sim 3 \%$ & $\sim 10 \%$ & Wii remote & DTW \\
\hline Bailador et al.[2] & 96 & $1.8 \% \sim 2.1 \%$ & $\sim 5 \%$ & smartphone & DTW, Bayes, HMM \\
\hline Bashir et al.[3] & 40 & $\sim 1.8 \%$ & N/A & custom digital pen & DTW \\
\hline Chan et al.[6] & 16 & $0.8 \%$ & N/A & Leap Motion & random forest \\
\hline Tian et al.[31] & 18 & $\sim 2 \%$ & N/A & Kinect & DTW \\
\hline
\end{tabular}

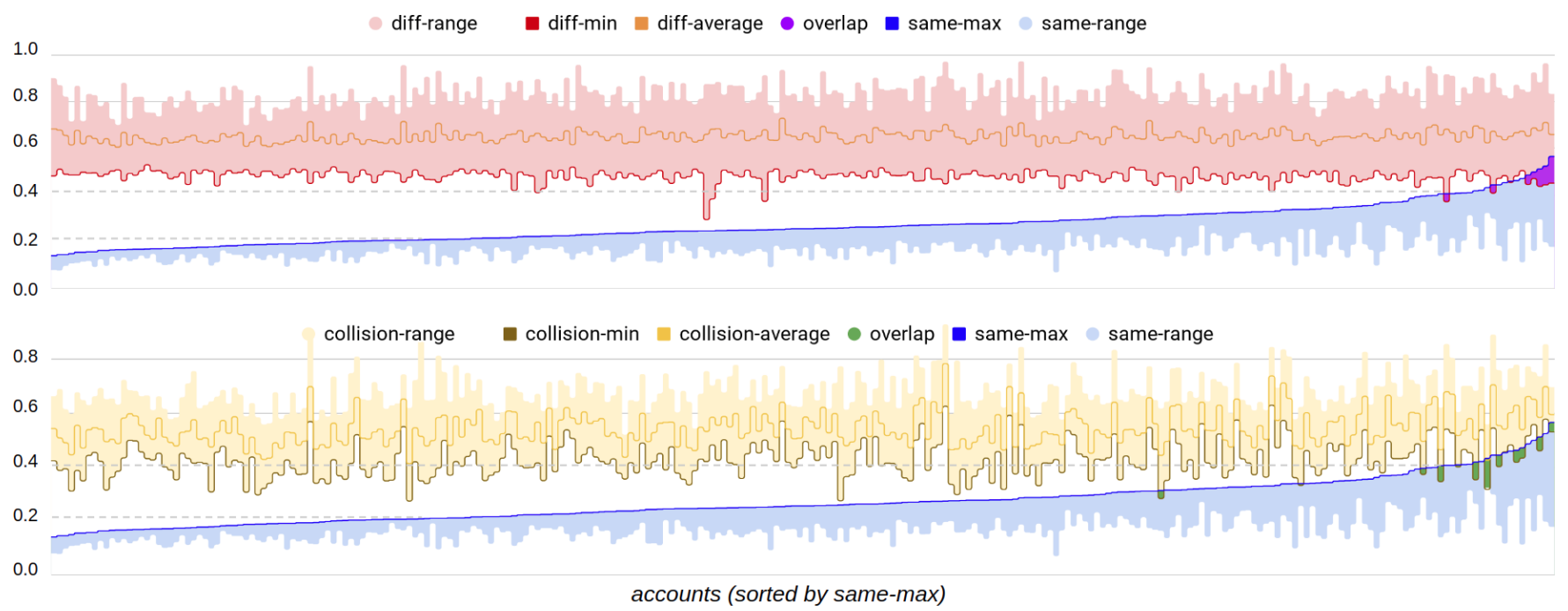

Figure 9: Matching score distribution of each account with the Feature Fusion method.

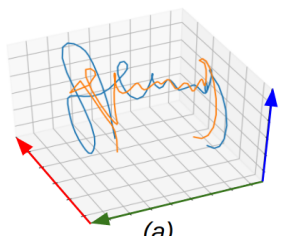

(a)

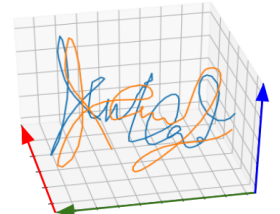

(b)

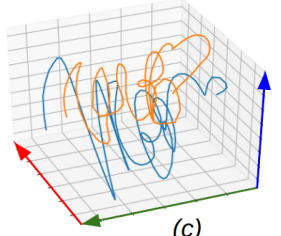

(c)

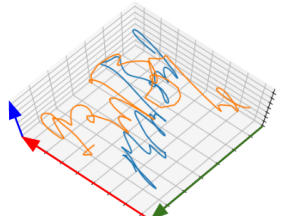

(d)

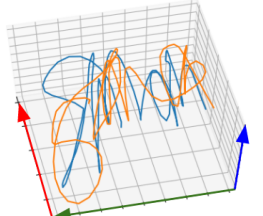

(e)

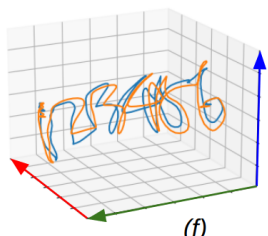

(f)

Figure 10: Examples of hard cases. The templates are in blue, and the testing signals are in orange.

two types of sensors use fundamentally different motion tracking methods. Our signal model and preprocessing steps remove the sensor-dependent information. As a result, we believe our feature analysis and authentication framework can also apply to a broad range of devices. Still, there are small differences between the results of different devices. For example, the signal quality is slightly better with the glove device, and the user behavior is slightly more consistent. This may be the result of the lack of field-of-view restriction with the glove device. Also, the glove device tracks the tip of the index finger, which moves more significantly than the center of the palm tracked by the camera device. The hand movement is more stable if a user writes it more naturally with the "muscle memory" rather than attention.

\subsection{Analysis of Hard Cases}

Besides collective performance results, in Figure 9, we also show the ranges of distance scores $\delta$ of the signals in different classes using the Feature Fusion method. On the top half of this figure, we show the ranges of distance scores for signals with $c=$ same and signals with $c=$ diff. Here, the two lines for "diff-min" and "diffavg" are the minimum and average of the distance scores for those login request signals with class label $c=$ diff for each account. The colored area "diff-range" shows the range of these distance scores. Similarly, "same-max" is the maximum of the distance scores for those login request signals with class label $c=$ same for each account, and the range of these scores is shown by the colored area "samerange". The intersection between the "diff-range" and "same-range" 
is shown in darker colors and denoted as "overlap". On the bottom half of this figure, we show the ranges of distance scores for signals with $c=$ same and signals with $c=$ collision in a similar way. The accounts are sorted by the "same-max" to show a trend from the "easy cases" on the left to the "hard cases" on the right.

There are a few testing signals with $c=$ diff which have smaller matching scores below a "reasonable threshold" (around 0.4, where FAR is $\approx 0.001 \%$ and FRR is $\approx 1 \%$ without considering collision). They are the first type of "hard cases," which significantly influence the security strength of the authentication system and further determine the threshold that should be used in practice. Even if these passcode strings are different in content, they may have similar stroke patterns when writing in the air because of the transitioning strokes. For example, there are two strings "feng" and "yang", and these signals are very similar because of the similarity in content and writing style, as shown in Figure 10 (a). This pair of signals and template has a matching distance score of 0.294. Another example is shown in Figure 10 (b), where the two strings are "Kaicheng" and "Kirkland". This pair of signals and template has a matching distance score of 0.373 .

On the rightmost of the figure, there are signals with $c=$ same with large matching distance scores above the reasonable threshold. They are the second type of hard cases which influence usability. Two examples of the hard cases are shown here. For Figure 10 (c), the signal and the template are generated by the same user writing the same passcode content "Victor", but the user changes the writing behavior, and the resulting $\delta$ is 0.524 . For Figure 10 (d) the signal and the template are generated by the same user writing the same passcode content "harrison67", and the resulting $\delta$ is 0.566 . In this example, at the end of the signal, many meaningless hand movements cause interference in the the alignment and the hand pose normalization in the signal preprocessing step 2. As a result, the scores of these hard cases are even larger than the typical distance score between a template and a signal with $c=$ collision. Such a collision example is shown in Figure 10 (e), where both the user and the imposter wrote "Simon" and the resulting distance score $\delta$ is 0.555 . On the contrary, in Figure 10 (f), we show a template and a signal obtained by the same user writing " 123456 ", and the distance score $\delta$ is only 0.187 .

\subsection{Spoofing Attack Analysis}

We collected a dataset with active spoofing attacks with both leakage of passcode content and visual leakage of passcode writing behavior. The dataset is similar the dataset 2, and we asked $10 \mathrm{im}$ postors to write the passcode of 180 accounts created by 90 users in the first dataset for five times. The impostors were informed of the content of the passcode, and they were allowed to see a video recording of the original account owner writing the passcode from the frontal side with unlimited times at any speed. We also briefly trained the imposters and paid them with more incentive money so as to ask them to put more effort in imitating the in-air-handwriting of the original users. There were 90 users in this spoofing attack experiment because only these 90 users granted permissions to us for recording the videos.

The results are shown in Table 3. Compared with the results with collision in Table 1, the performance of our framework under
Table 3: Evaluation results with active spoofing.

\begin{tabular}{|c|c|c|c|c|c|}
\hline method & EER & FAR1K & FAR10K & ZeroFAR & FRR100 \\
\hline DTW (camera) & 4.7 & 59.3 & 78.3 & 78.3 & 24.5 \\
\hline T-Fusion (camera) & 2.9 & 26.2 & 33.1 & 33.1 & 12.1 \\
\hline DTW (glove) & 3.5 & 19.6 & 52.7 & 52.7 & 17.2 \\
\hline T-Fusion (glove) & 1.9 & 9.8 & 37.1 & 37.1 & 10.5 \\
\hline
\end{tabular}

active spoofing attacks does not collapse completely. The impostors reported that when the user writes the passcode fast like a signature, it was difficult to mimic the behavior. However, there were still a significant number of accounts that can be successfully cracked by the impostors if the distance score threshold is set to a practical value such that the FRR is close to $1 \%$, as shown in the column "FRR100" (at this threshold, the FAR is approximately between $10^{-4}$ to $10^{-5}$ without considering collision). As a result, we believe our framework is more like a password-based authentication system where the content of the passcode should be kept as a secret, rather than a signature-based authentication system. On the other hand, a password-based authentication system will not be able to defend any unauthorized login if the password is leaked to the attackers, but our framework can still provide a certain level of defense such that only about $10 \%$ to $25 \%$ percent of unauthorized login attacks are successful under visual leakage of the passcode content.

\subsection{Persistence Analysis}

We collect an additional dataset to analyze the persistence of the in-air-handwriting passcode. 40 users participating in the dataset 1 in section 3.3 were asked to write their passcodes five times as a session, and 10 sessions in total. The data from each session is collected at a different day. Then, we apply the step 2 of the experiment protocol using the data of each session and the T-Fusion algorithm. The score variation among the ten sessions is shown in Figure 11. Each column of this plot corresponds to the results in one session, which is similar to the score histogram shown in Figure 9. Here, the scores of all 40 accounts are plotted as scattered points from left to right across the width of the column for the session. There is an increase of the scores of the login requests of legitimate users over time if the templates and the classifiers are not updated, although the extent of increase slows down after the fourth session. However, the scores of the login requests of the random guessing attacks do not have noticeable changes. These two factors make the system harder to distinguish legitimate users from attackers, which eventually leads to more frequent false rejection of legitimate users if the decision threshold is not changed, shown in Figure 12.

There are two reasons. First, there are inherent variations of user behaviors, which leads to differences in signals even for the same user writing the same string. For example, most users write from left to right using the index finger, but this left-to-right movements of hand usually do not have a fixed angle relative to the pointing direction of the index finger when writing the same content every time in the air. The variation generally grows with multiple sessions. However, the xyz-axes are determined by the pointing direction in the signal preprocessing steps, which leads to variations in the signals. This is more significant for the camera device because it directly tracks position and orientation. Second, the authentication algorithm is not perfect. There are limited amount of 

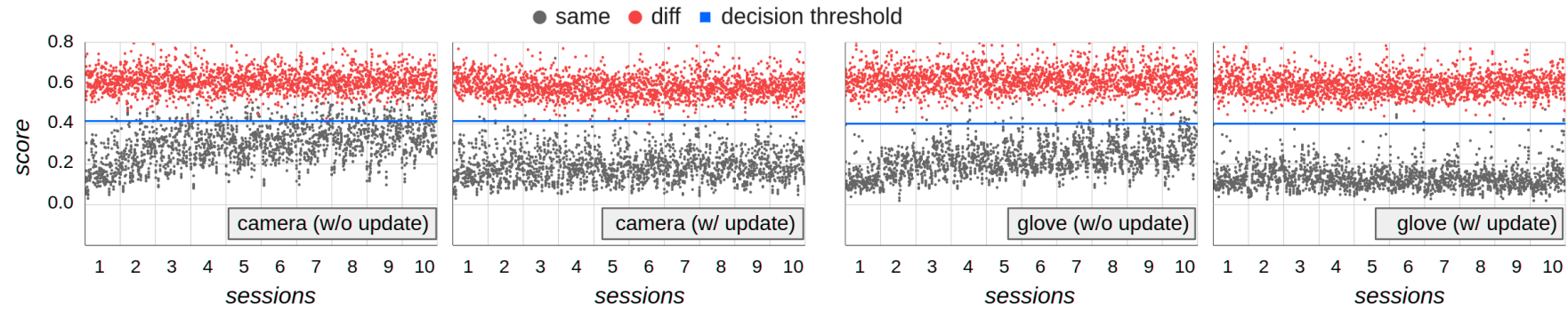

Figure 11: Variation of scores of legitimate user login requests and random guessing attacks among ten sessions. The decision threshold is set to 0.413 for the camera device and 0.401 for the glove device, such that the corresponding single session performance metrics are $\mathrm{FRR} \approx 1 \%, \mathrm{FAR} \approx 0.001 \%$ without considering collision, and FAR $\approx 12 \%$ with considering collision.
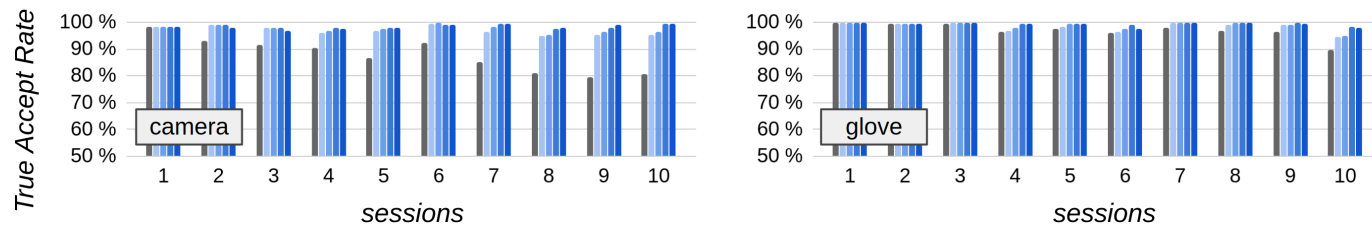

w/o update

w/ update (first session)

w/ update (first two sessions)

w/ update (first five sessions)

w/ update (ten sessions)

Figure 12: Variation of True Acceptance Rate (TAR, i.e., 1 - FRR) among ten sessions using the decision threshold in Fig. 11.

signals obtained at registration, which make the system difficult to capture or predict the long-term in-air-handwriting behavior variation. Meanwhile, the classification is designed to utilize signal-level differences directly for more effective detection of spoofing attacks from legitimate login requests, which also limits its generalization capability under long-term variation.

To accommodate these variations, one solution is asking the users to update the templates and retrain the SVM classifier. The updated template is a combination of an old template and the updating signal, i.e., $T \leftarrow(1-\lambda) T+\lambda S$, similar as [19]. After the updating, the SVM classifier is retrained using the old templates and the updating signals as positive data points and templates of other accounts as negative data points. In Figure 12, we show the performance change with four different scenarios: (a) only do the updating in the first session while not changing the templates and the SVM classifier after that; (b) similar to (a) but do the updating in both the first session and the second session; (c) similar to (a) but do the updating in the first five sessions; (d) do the updating in all ten sessions, and this is the scenario that generates the results labeled with update in Figure 11. From the results, we can observe that long term performance can be improved by the updating, even only updating at the first login immediately after registration can boost the true accept rate to above $95 \%$ for all remaining sessions.

\section{CONCLUSIONS AND FUTURE WORK}

This paper presents a user authentication framework with two different types of devices using various features of in-air-handwriting passcode, including temporal features, statistical features, and hand geometry features. We also conduct a comparative evaluation of various matching algorithms and an analysis of these features to show the insights of the algorithms. Although the results are promising, it is still in an early stage with limitations. For example, the tracking performance of the camera device degrades when the hand moves fast or in some poses. Also, there might be usability concerns for asking the user to put on a glove unless the glove is a default gesture input device of the computing platform. Hence, we plan to design a handheld device with localization capability or a lightweight ring-like device with the same inertial sensor as the glove in the future. Moreover, our matching algorithm and persistence study is primitive. It is not clear whether freestyle in-air-handwriting passcode is stable enough for user identity verification in the long term under various environment. We plan to collect a large dataset of in-air-handwriting of all possible combination of characters and develop a data-driven method to convert a signal to a sequence of stroke representations for fine-grained matching. Meanwhile, we plan to collect data from the same user with more sessions and expand the time span. Constraints in the writing content or visual stimuli can also be added in future experiments to make the users' behavior more stable. Furthermore, we present a primitive usability study in Appendix B with a survey of 100 users, but the responses of the survey are subjective to each user and the evaluation is qualitative. Hence, we intend to collect objective and quantitative metrics of the usability in the future. Still, we believe such an in-air-handwriting passcode-based authentication method has a great potential in many wearable and VR/AR applications in the future, and we hope our datasets can be helpful to researchers in this field.

\section{REFERENCES}

[1] Ilhan Aslan, Andreas Uhl, Alexander Meschtscherjakov, and Manfred Tscheligi. 2014. Mid-air authentication gestures: an exploration of authentication based on palm and finger motions. In Proceedings of the 16th International Conference on Multimodal Interaction. ACM.

[2] Gonzalo Bailador, Carmen Sanchez-Avila, Javier Guerra-Casanova, and Alberto de Santos Sierra. 2011. Analysis of pattern recognition techniques for in-air signature biometrics. Pattern Recognition (2011).

[3] Muzaffar Bashir and Jurgen Kempf. 2009. Person authentication with RDTW based on handwritten PIN and signature with a novel biometric smart pen device. In IEEE Workshop on Computational Intelligence in Biometrics: Theory, Algorithms, 
and Applications, 2009. CIB 2009. IEEE, 63-68.

[4] Donald J Berndt and James Clifford. 1994. Using Dynamic Time Warping to Find Patterns in Time Series.. In KDD workshop, Vol. 10. Seattle, WA.

[5] Aman Chahar, Shivangi Yadav, Ishan Nigam, Richa Singh, and Mayank Vatsa 2015. A Leap Password based verification system. In IEEE 7th International Conference on Biometrics Theory, Applications and Systems (BTAS). IEEE.

[6] Alexander Chan, Tzipora Halevi, and Nasir Memon. 2015. Leap Motion Controller for Authentication via Hand Geometry and Gestures. In International Conference on Human Aspects of Information Security, Privacy, and Trust.

[7] Gradeigh D Clark and Janne Lindqvist. 2015. Engineering Gesture-based Authentication Systems. IEEE Pervasive Computing (2015).

[8] Elisabetta Farella, Sile O’Modhrain, Luca Benini, and Bruno Riccó. 2006. Gesture signature for ambient intelligence applications: a feasibility study. In PerCom Springer.

[9] Julian Fierrez, Aythami Morales, Ruben Vera-Rodriguez, and David Camacho. 2018. Multiple classifiers in biometrics. Part 1: Fundamentals and review. Information Fusion (2018).

10] Marta Gomez-Barrero, Javier Galbally, Aythami Morales, Miguel A Ferrer, Julian Fierrez, and Javier Ortega-Garcia. 2014. A novel hand reconstruction approach and its application to vulnerability assessment. Information Sciences (2014).

[11] Feng Hong, Meiyu Wei, Shujuan You, Yuan Feng, and Zhongwen Guo. 2015 Waving authentication: your smartphone authenticate you on motion gesture. In Proceedings of the 33rd Annual ACM Conference Extended Abstracts on Human Factors in Computing Systems. ACM.

[12] Donato Impedovo and Giuseppe Pirlo. 2008. Automatic signature verification The state of the art. IEEE Transactions on Systems, Man, and Cybernetics, Part C (Applications and Reviews) 38, 5 (2008), 609-635.

[13] Donato Impedovo and Giuseppe Pirlo. 2018. Automatic signature verification in the mobile cloud scenario: survey and way ahead. IEEE Transactions on Emerging Topics in Computing (2018).

[14] Ajay Kumar, David CM Wong, Helen C Shen, and Anil K Jain. 2003. Personal verification using palmprint and hand geometry biometric. In International Conference on Audio-and Video-Based Biometric Person Authentication. Springer.

[15] Jonathan Lester, Blake Hannaford, and Gaetano Borriello. 2004. "Are You with Me?"-Using Accelerometers to Determine If Two Devices Are Carried by the Same Person. In PerCom. Springer.

[16] Jaime Lien, Nicholas Gillian, M Emre Karagozler, Patrick Amihood, Carsten Schwesig, Erik Olson, Hakim Raja, and Ivan Poupyrev. 2016. Soli: Ubiquitous gesture sensing with millimeter wave radar. ACM Transactions on Graphics (TOG) 35, 4 (2016), 142.

[17] Jiayang Liu, Lin Zhong, Jehan Wickramasuriya, and Venu Vasudevan. 2009. uWave: Accelerometer-based personalized gesture recognition and its applications. Pervasive and Mobile Computing (2009).

[18] Duo Lu. 2021. 3D In-Air-Handwriting based User Login and Identity Input Method. Ph.D. Dissertation. Arizona State University.

[19] Duo Lu, Dijiang Huang, Yuli Deng, and Adel Alshamrani. 2018. Multifactor User Authentication with In-Air-Handwriting and Hand Geometry. In 2018 International Conference on Biometrics (ICB). IEEE.

[20] Duo Lu, Dijiang Huang, and Anshul Rai. 2019. FMHash: Deep Hashing of In-Air Handwriting for User Identification. (2019).

[21] Duo Lu, Kai Xu, and Dijiang Huang. 2017. A Data Driven In-Air-Handwriting Biometric Authentication System. In IFCB. IEEE.

[22] Alessandra Lumini and Loris Nanni. 2017. Overview of the combination of biometric matchers. Information Fusion (2017).

[23] Rene Mayrhofer and Hans Gellersen. 2007. Shake well before use: Authentication based on accelerometer data. In PerCom. Springer.

[24] Ben Nassi, Alona Levy, Yuval Elovici, and Erez Shmueli. 2016. Handwritten Signature Verification Using Hand-Worn Devices. arXiv:1612.06305 (2016)

[25] George E Raptis, Christina Katsini, Andrew Jian-Lan Cen, Nalin Asanka Gam agedara Arachchilage, and Lennart E Nacke. 2021. Better, Funner, Stronger: A Gameful Approach to Nudge People into Making Less Predictable Graphical Password Choices. In Proceedings of the 2021 CHI Conference on Human Factors in Computing Systems. 1-17.

[26] Napa Sae-Bae, Nasir Memon, Katherine Isbister, and Kowsar Ahmed. 2014. Multitouch gesture-based authentication. IEEE transactions on information forensics and security 9, 4 (2014), 568-582.

[27] Napa Sae-Bae, Jonathan Wu, Nasir Memon, Janusz Konrad, and Prakash Ishwar 2019. Emerging NUI-Based Methods for User Authentication: A New Taxonomy and Survey. IEEE Transactions on Biometrics, Behavior, and Identity Science (2019).

[28] Hasan Sajid and S Cheung Sen-ching. 2015. VSig: Hand-gestured signature recognition and authentication with wearable camera. In International Workshop on Information Forensics and Security (WIFS). IEEE

[29] Raul Sanchez-Reillo, Carmen Sanchez-Avila, and Ana Gonzalez-Marcos. 2000. Biometric identification through hand geometry measurements. IEEE Transactions on pattern analysis and machine intelligence (2000).

[30] Xiaoyuan Suo, Ying Zhu, and G Scott Owen. 2005. Graphical passwords: A survey. In 21st Annual Computer Security Applications Conference (ACSAC'05) IEEE, 10-pp.

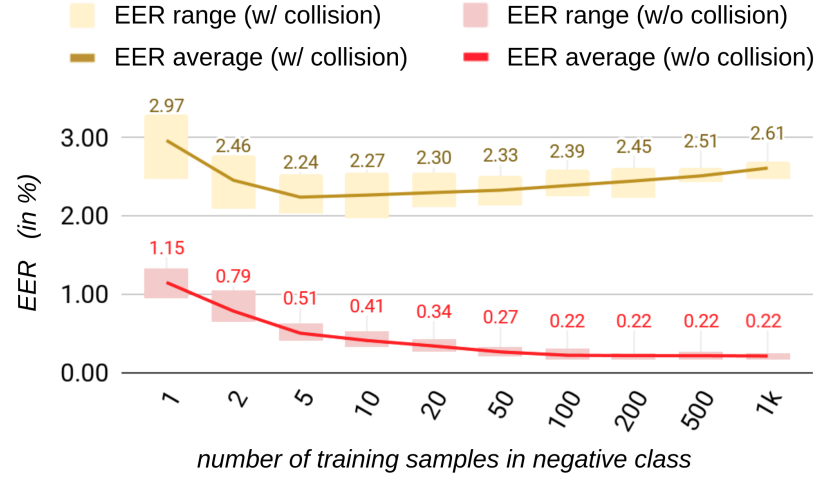

Figure 13: Performance change of the T-Fusion method with respect to the number of negative training samples.

[31] Jing Tian, Chengzhang Qu, Wenyuan Xu, and Song Wang. 2013. KinWrite: Handwriting-Based Authentication Using Kinect.. In NDSS.

[32] Ruben Tolosana, Ruben Vera-Rodriguez, Julian Fierrez, and Javier Ortega-Garcia. 2021. DeepSign: Deep on-line signature verification. IEEE Transactions on Biometrics, Behavior, and Identity Science 3, 2 (2021), 229-239.

[33] Frank Weichert, Daniel Bachmann, Bartholomäus Rudak, and Denis Fisseler. 2013. Analysis of the accuracy and robustness of the leap motion controller. Sensors 13, 5 (2013), 6380-6393.

[34] Susan Wiedenbeck, Jim Waters, Jean-Camille Birget, Alex Brodskiy, and Nasir Memon. 2005. PassPoints: Design and longitudinal evaluation of a graphical password system. International journal of human-computer studies 63, 1-2 (2005), 102-127.

[35] Jonathan Wu, James Christianson, Janusz Konrad, and Prakash Ishwar. 2015. Leveraging shape and depth in user authentication from in-air hand gestures. In International Conference on Image Processing (ICIP). IEEE.

[36] Jonathan Wu, Janusz Konrad, and Prakash Ishwar. 2013. Dynamic time warping for gesture-based user identification and authentication with Kinect. In International Conference on Acoustics, Speech and Signal Processing. IEEE.

[37] Yulong Yang, Gradeigh D Clark, Janne Lindqvist, and Antti Oulasvirta. 2016. Free-form gesture authentication in the wild. In Proceedings of the $2016 \mathrm{CHI}$ Conference on Human Factors in Computing Systems. 3722-3735.

[38] Alexandros Zaharis, Adamantini Martini, Panayotis Kikiras, and George Stamoulis. 2010. User authentication method and implementation using a three-axis accelerometer. In International Conference on Mobile Lightweight Wireless Systems.

\section{APPENDICES}

\section{A ANALYSIS ON FEATURE FUSION}

One question is whether the weights learned from other accounts only work for those accounts in T-Fusion and S-Fusion instead of general random guessing attacks. To answer this, we conduct another set of experiments where fewer randomly selected negative training samples are used to learn the weights at registration. The results are shown in Figure 13. The performance degrades slightly with less negative training sample. However, even if signals from most of the other accounts are not observed in training, the matching algorithm can still work. One possible explanation is that the element-wise distances are generally large for negative training samples, and they form similar cluster patterns in high dimensional feature space as Figure 7. Hence, a linear maximum margin decision plane fitted from training data (i.e., SVM) is relatively robust with the variation of the cluster shape. This also explains why we can obtain reasonably good performance with only five positive training samples. We believe this is the advantage of template matching based methods since the system only needs to calculate a rough 


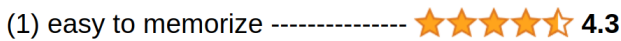

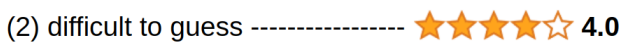

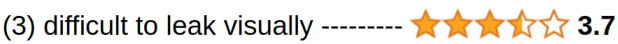
(4) difficult to mimic on leakage -- the 4.0

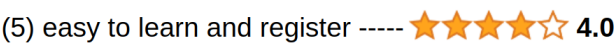

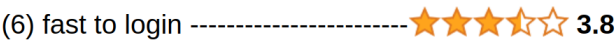
(7) easy to update and revoke ---- the

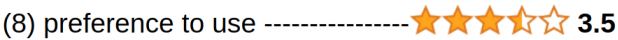

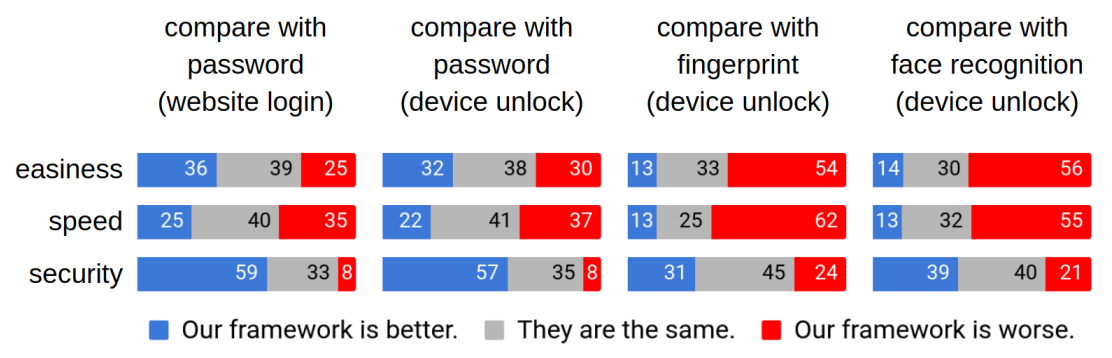

Figure 14: User evaluation results.

decision boundary relative to the template (which is generally easy) rather than learning a model that must "remember" the templates as in Hidden Markov Model or deep neural network-based methods (which is generally hard). In fact, we tried a deep neural network, but the performance is inferior. However, since the templates are stored and retrieved in alignment, they are similar to plain text passwords and cannot be hashed.

\section{B USER EVALUATION}

We investigated the usability of our framework by taking questionnaire from 100 users participated in the collection of dataset 1 . Each user is asked to submit a questionnaire.

First, the users evaluate various aspects of our in-air-handwriting based login framework by putting down a score from 1 (strongly disagree) to 5 (strongly agree) on the following statements. In these statements, "the user" refers to the user taking this questionnaire. The users are required to provide these scores based on their subjective feeling.

(1) Since the passcode string is created by the user, the passcode content is easy to memorize.

(2) From the perspective of a stranger, the passcode content created by the user is difficult to guess.

(3) The passcode content is difficult to leak visually, i.e., it is difficult to guess the passcode content based on the in-airhandwriting movements.

(4) From the perspective of an imposter, it is difficult to mimic the in-air-handwriting movements after seen them.

(5) It is easy to learn and register with our in-air-handwriting based login framework.

(6) It is fast to login through our in-air-handwriting based login framework

(7) It is easy to update or revoke the passcode if the user forgets it or if the user wants to change it.

(8) The user prefers to use it as the primary login method through a gesture input interface.

The results are shown in Figure 14 (left). Overall, users feel positive regarding our framework, although the usability is not exceptionally good in a few aspects.

Second, we ask the user to compare our framework with the password-based systems and biometrics including fingerprint and face. The comparison is made on three aspects, the easiness of usage, login speed, and security based on the feeling of users. The user has three options: (a) our framework is better, (b) both methods are the same, or it is difficult to decide which one is better or worse, (c) our framework is worse. The results are shown in Figure 14 (right). We can see that the user has a mixed attitude on the usability compared to traditional password, but our framework can not replace biometrics like fingerprint and face for device unlock. However, the majority of the users feel that our framework is more secure than traditional password, and more than half of them feel it is more secure than fingerprint and face.

Third, we ask the following questions:

1) Compared to password, our framework considers both handwriting content and handwriting style, i.e., combining a password and a biometric trait. Is this feature important for a user authentication system?

2) Compared to biometrics, our framework allows change and revoke the in-air-handwriting passcode, which is unlinked to personal identity. Is this feature important for a user authentication system?

Among the surveyed users, $89 \%$ and $82 \%$ of them answer "important" for the first and second features respectively. Combined with the previous results, we can conclude that our framework does not intend to replace existing password-based solution or biometrics. Instead, due to its unique features that passwords and biometrics lack, we believe that our framework is suitable in scenarios where such features matter and where passwords and biometrics are not applicable, for example, login over gesture interface on VR headset or in operating theater.

At last, we ask the user which type of device is preferred between a wearable device and a contactless device for hand motion tracking. $21 \%$ of the users choose the wearable device and the other $79 \%$ choose the contactless device. 